\title{
Does diabetes impact therapeutic immunomodulation therapy decisions for kidney transplant recipients? Data from the Folic Acid for Vascular Outcome Reduction in Transplant (FAVORIT) trial
}

This article was published in the following Dove Press journal: International Journal of Nephrology and Renovascular Disease 18 August 2017

Number of times this article has been viewed

\author{
Larry A Weinrauch ${ }^{1-4}$ \\ John A D'Elia ${ }^{2-4}$ \\ Matthew R Weir ${ }^{5}$ \\ Suphamai Bunnapradist ${ }^{6}$ \\ Peter Finn' \\ Jiankang Liu' \\ Brian Claggett ${ }^{\prime}$ \\ Anthony P Monaco 3,4 \\ 'Cardiovascular Division, Brigham \\ and Women's Hospital, Boston, \\ MA, ${ }^{2}$ Kidney and Hypertension \\ Section, Joslin Diabetes Center, \\ Boston, MA, ${ }^{3}$ Medicine and Surgery, \\ Beth Israel Deaconess Medical Center \\ Boston MA, ${ }^{4}$ Harvard Medical School, \\ Boston MA, ${ }^{5}$ University of Maryland \\ School of Medicine, Baltimore, MD, \\ ${ }^{6}$ Division of Nephrology, Department \\ of Medicine University of California at \\ Los Angeles, Los Angeles, CA, USA
}

Correspondence: Larry A Weinrauch 52I Mount Auburn Street, Suite 204, Watertown, MA 02472, USA

Email lweinrauch@hms.harvard.edu

\begin{abstract}
Although survival has improved for kidney transplant recipients over the past several decades, long-term survival in diabetic cohorts still is significantly less than that of non-diabetic cohorts. We hypothesized that among stable kidney transplant recipients, there might be differences between subgroups with and without diabetes with respect to prevalence of prior cardiovascular events and post-transplant antihypertensive and immunosuppressive therapy. We performed a post hoc analysis of participants in the Folic Acid for Vascular Outcome Reduction in Transplant (FAVORIT) trial, a multicenter international trial of 4110 prevalent kidney transplant recipients enrolled from 2002 to 2007 evaluating the effect of homocysteine-lowering vitamin therapy on cardiovascular outcomes. There were 2447 participants without diabetes, 166 with type 1 diabetes, and 1447 with type 2 diabetes at study entry, which occurred on average 4 years post-transplant. Recipients with diabetes had a greater prevalence of prior cardiovascular events, were more likely to have required multiple medications to control hypertension, and were more likely to have received tacrolimus as opposed to cyclosporine than the non-diabetic transplant recipients (all $p<0.001$ ). The effect of differences in treatment of non-diabetic vs diabetic cohorts after stable renal transplantation upon outcomes has not yet been studied and could provide additional information that might lead to improved care.
\end{abstract}

Keywords: diabetes, immunomodulation, kidney transplant, immunosuppression

\section{Introduction}

In the US, the latest statistics has revealed (2014-USRDS/UNOS) that more than 17,000 kidney transplants are done annually. Twice as many allografts come from deceased donors as from live donors. Diabetes mellitus is the cause of end-stage renal disease in $40 \%$ of this transplant population. Tens of thousands of diabetic patients have benefited from renal transplantation since the demonstration in 1970s that prescreening of potential recipients could result in patient and allograft survivals for diabetic patients similar to those of non-diabetic patients over the first 2 years. ${ }^{1-3}$ Indeed, such success has been extended to some patients with preexistent heart disease by the early detection and treatment of coronary arterial disease. ${ }^{4}$ Although diabetic populations may now achieve similar transplant benefits to non-diabetic populations, long-term overall outcomes remain poor.

We had the opportunity to analyze data from a large population $(n=4110), 40 \%$ of whom had diabetes. All studied subjects had successful renal transplantation and 
were considered clinically stable. We hypothesized that among kidney transplant recipients, there might be differences between subgroups with and without diabetes with respect to prevalence of prior cardiovascular events (PCVE) and post-transplant antihypertensive and immunosuppressive therapies. Such differences, should they exist, could be expected to relate to both allograft source and recipient factors and, therefore, impact and confound choices of therapy. Subgroup analysis may be clinically useful to improve longterm survival.

\section{Materials and methods}

This is a retrospective analysis of the data set from the international Folic Acid for Vascular Outcome Reduction in Transplant (FAVORIT) trial, which was designed to determine whether a combination of vitamins B6, B12, and folic acid would reduce cardiovascular end points in a large renal transplant recipient cohort. The full methods and results of this trial are reported elsewhere. ${ }^{5,6}$ Enrollment involved 4110 patients in 27 clinical sites including data from the baseline visit from August 2002 through January 2007. All subjects were between 35 and 75 years of age, had elevated homocysteine levels $(>11 \mathrm{~mol} / \mathrm{L}$ for women and $>12 \mathrm{~mol} / \mathrm{L}$ for men), and at least 6 months after kidney transplant had stable kidney function. Follow-up contacts occurred every 6 months through January 31, 2010, to obtain study-related outcomes through June 24, 2009.

Subjects were randomized and classified as non-diabetic and type 1 or type 2 diabetic study subjects. Patients were similarly classified by the presence or absence of cardiovascular diagnoses prior to randomization. Demographic information is available in Table 1 . There were 2447 non-diabetic, 166 type 1 diabetic and 1497 type 2 diabetic study subjects. Laboratory tests and medication use are reported from the baseline visit prior to randomization. Prescription medica-

Table I Baseline demographics by DM types (DMI, DM2, and non-diabetes)

\begin{tabular}{|c|c|c|c|c|}
\hline Baseline at enrollment & $\begin{array}{l}\text { No DM } \\
(\mathrm{N}=2447)\end{array}$ & $\begin{array}{l}\text { DMI } \\
(N=166)\end{array}$ & $\begin{array}{l}\text { DM2 } \\
(\mathrm{N}=\mid \text { | 497) }\end{array}$ & $p$-value \\
\hline Age & $51.32 \pm 9.52$ & $51.49 \pm 9.29$ & $52.96 \pm 9.24$ & $<0.001$ \\
\hline Non-White & $513(21.4 \%)$ & 31 (19.0\%) & $40 \mathrm{I}(27.4 \%)$ & $<0.001$ \\
\hline Country & & & & $<0.001$ \\
\hline US & 1667 (55.6\%) & $120(4 \%)$ & $1213(40.4 \%)$ & \\
\hline Canada & 346 (69.5\%) & $20(4 \%)$ & 132 (26.5\%) & \\
\hline Brazil & 434 (70.9\%) & $26(4.3 \%)$ & $152(28.8 \%)$ & \\
\hline Female & $921(37.6 \%)$ & $54(32.5 \%)$ & $553(36.9 \%)$ & 0.41 \\
\hline Smoker (current) & 297 (12.1\%) & 25 (I5.1\%) & $129(8.6 \%)$ & $<0.001$ \\
\hline Prevalent CVD (\%) & $324(13.3 \%)$ & $52(31.3 \%)$ & $444(29.7 \%)$ & $<0.001$ \\
\hline Prevalent hypertension (\%) & $2225(91.0 \%)$ & $146(88.0 \%)$ & 1407 (94.1\%) & $<0.001$ \\
\hline Graft vintage (years) & $4.2[1.7,7.9]$ & $2.9[1.3,7.1]$ & $3.8[1.6,7.1]$ & 0.013 \\
\hline BMI & $28.59 \pm 5.94$ & $22.79 \pm 2.21$ & $30.84 \pm 6.39$ & $<0.001$ \\
\hline Creatinine $(\mu \mathrm{mol} / \mathrm{L})$ & $144.59 \pm 42.30$ & $139.56 \pm 38.85$ & $|44.34 \pm 4| .99$ & 0.33 \\
\hline eGFR & $48.6 I \pm|5.9|$ & $51.06 \pm 17.69$ & $48.79 \pm 16.47$ & 0.17 \\
\hline CKD & & & & 0.23 \\
\hline $\mathrm{GFR} \geq 90 \mathrm{~mL} / \mathrm{min}$ & $42(1.8 \%)$ & $5(3.1 \%)$ & $22(1.5 \%)$ & \\
\hline $60 \leq \mathrm{GFR}<90 \mathrm{~mL} / \mathrm{min}$ & $466(19.5 \%)$ & $38(23.3 \%)$ & $315(21.5 \%)$ & \\
\hline $30 \leq \mathrm{GFR}<60 \mathrm{~mL} / \mathrm{min}$ & $1659(69.3 \%)$ & 110 (67.5\%) & $969(66.2 \%)$ & \\
\hline $15 \leq \mathrm{GFR} 8<30 \mathrm{ml} / \mathrm{min}$ & 227 (9.5\%) & $10(6.1 \%)$ & $157(10.7 \%)$ & \\
\hline $\mathrm{GFR}<15 \mathrm{~mL} / \mathrm{min}$ & $\mathrm{I}(0.0 \%)$ & $0(0.0 \%)$ & $0(0.0 \%)$ & \\
\hline Cholesterol (mmol/L) & $4.91 \pm 1.15$ & $4.46 \pm 0.98$ & $4.61 \pm 1.12$ & $<0.001$ \\
\hline Triglycerides (mmol/L) & $2.24 \pm 1.45$ & $1.73 \pm 1.00$ & $2.32 \pm 2.84$ & 0.004 \\
\hline HDL cholesterol $(\mathrm{mmol} / \mathrm{L})$ & $1.20 \pm 0.35$ & $1.30 \pm 0.46$ & $1.18 \pm 0.36$ & $<0.001$ \\
\hline LDL cholesterol (mmol/L) & $2.7 I \pm 0.90$ & $2.36 \pm 0.76$ & $2.44 \pm 0.82$ & $<0.001$ \\
\hline Homocysteine $(\mu \mathrm{mol} / \mathrm{L})$ & $16.11 \pm 7.89$ & $15.15 \pm 4.58$ & $15.93 \pm 6.24$ & 0.51 \\
\hline \multicolumn{5}{|l|}{ Cardiovascular history } \\
\hline $\mathrm{MI} / \mathrm{CHD}$ & $225(9.2 \%)$ & $37(22.3 \%)$ & 304 (20.3\%) & $<0.001$ \\
\hline Stroke/CBVD & $109(4.5 \%)$ & $18(10.8 \%)$ & $144(9.6 \%)$ & $<0.001$ \\
\hline AAA repair/leak & $45(1.8 \%)$ & $9(5.4 \%)$ & $109(7.3 \%)$ & $<0.001$ \\
\hline CVD (any) & 324 (13.3\%) & $52(31.3 \%)$ & 444 (29.7\%) & $<0.001$ \\
\hline Renal revascularization & $77(3.2 \%)$ & $15(9.0 \%)$ & $129(8.6 \%)$ & $<0.001$ \\
\hline Amputation & $33(1.3 \%)$ & $7(4.2 \%)$ & $70(4.7 \%)$ & $<0.001$ \\
\hline
\end{tabular}

Abbreviations: DM, diabetes mellitus; DMI, type I diabetes mellitus; DM2, type 2 diabetes mellitus; CVD, cardiovascular disease; BMI, body mass index; eGFR, estimated glomerular filtration rate; CKD, chronic kidney disease; HDL, high-density lipoprotein; LDL, low-density lipoprotein; MI, myocardial infarction; CHD, congenital heart disease; AAA, abdominal aortic aneurysm; CVD, cardiovascular disease. 
tions taken regularly during the past month were recorded during participant interviews. Exclusion criteria included associated comorbidities that could be expected to limit survival (cancer, end-stage HIV, and hepatic, pulmonary, or cardiac disease) as well as recent ( $<3$ months) cardiovascular and renal events or surgical procedures.

History of cardiovascular events included the following classifications:

- Myocardial infarction or coronary artery revascularization (ie coronary artery bypass surgery or angioplasty)

- Stroke (thromboembolic or hemorrhagic)

- Carotid arterial revascularization (endarterectomy or angioplasty)

- Abdominal or thoracic aortic aneurysm repair

- Renal arterial revascularization (bypass surgery or angioplasty)

- Lower extremity arterial revascularization (bypass surgery or angioplasty)

- Lower extremity amputation above the ankle

Table 2 lists medications at baseline based on the absence or type of diabetes. Blood pressure-lowering medications included angiotensin converting enzyme inhibitors (ACEi), angiotensin receptor blockers (ARB), beta blockers, dihydropyridine calcium channel blockers, non-dihydropyridine calcium channel blockers, vasodilators, centrally active drugs, loop diuretics, and other diuretics. Lipid-lowering medications included 3-hydroxy-3-methyl-glutaryl-coenzyme A (HMG CoA) reductase inhibitors, bile acid sequestrants, niacin, gemfibrozil, fenofibrate, bezafibrate, clofibrate, probucol, and ezetimibe. Antiplatelet medications included aspirin, clopidogrel, ticlopidine, and other antiplatelet agents. Antidiabetic agents included insulin, sulfonylureas, rosiglitazone, pioglitazone, metformin, and other antidiabetic agents (Dipeptidyl peptidase-4 [DPP-4]inhibitors, Glucagon-like peptide-1 receptor [GLP-1] agonists). Transplant-specific immunosuppression regimens at baseline included cyclosporine A, tacrolimus, sirolimus, mycophenolate mofetil, azathioprine, and prednisone. Transplant immunosuppression medications were classed by mechanism of action: calcineurin inhibitors (cyclosporine A and tacrolimus), mechanistic target of rapamycin inhibitors (sirolimus), bone marrow suppressants (mycophenolate mofetil and azathioprine), and antilymphocyte agents (prednisone).

\section{Statistical analysis}

All baseline information is summarized using means and standard deviations or, for skewed variables, medians and interquartile ranges for continuous variables and using counts and percentages for dichotomous variables. In order to test for trends in baseline characteristics across categories of estimated glomerular filtration rate, linear regression, Cuzick's nonparametric trend test, and Cochran-Armitage test for trend were used. Global tests of equality are conducted using ANOVA, the Kruskal-Wallis test, and Pearson's chi-squared test, respectively. $p$-values $<0.05$ are considered significant. All analyses were conducted using STATA 13 (StataCorp LP, College Station, TX, USA).

\section{Results}

\section{Demographics}

As noted in Table 1, there was regional variation with respect to prevalence of type 2 diabetes (higher in the US compared to either Canada or Brazil). Age and transplant function were similar in the three groups. As would be expected, hypertension and significant cardiovascular history were much more prevalent in the diabetes cohorts. The diabetic transplant recipients were more than twice as likely to have survived a myocardial infarction, stroke, aneurysm repair, or amputation. Statin and aspirin use was higher when compared to the non-diabetic patient cohort, with lower lipid measurements. Decreased low-density lipoprotein and cholesterol were noted in the diabetic cohort, related to the excess of antecedent cardiovascular events. A high prevalence of current smoking in the type 1 diabetic transplant population compared to both non-diabetic and type 2 transplant diabetic populations confirms a prior observation from Warsaw, Poland, in an epidemiologic non-transplantation study. ${ }^{7}$ To our knowledge, the lower prevalence of tobacco smoking in type 2 diabetic transplant recipients when compared to a non-diabetic cohort has not been reported previously.

\section{Medication use at baseline}

Table 2 lists baseline medications for the three groups (nondiabetic and type 1 and type 2 diabetic groups).

- Although immunosuppressant combination therapy was similar among cohorts, increased use of tacrolimus with decreased use of cyclosporine was noted in the diabetic cohort when compared with the non-diabetic cohort.

- Antihypertensive medication use was increased in patients with type 2 (insulin-resistant) diabetes. Angiotensinactive drugs (ARB and ACEi) were used more in diabetic patients. Use of four or more antihypertensive medications was twice as high among the type 2 diabetic cohort as either type 1 or non-diabetic cohorts. 
Table 2 Baseline medications by DM types (DMI, DM2, and non-diabetes)

\begin{tabular}{|c|c|c|c|c|}
\hline Medications & $\begin{array}{l}\text { Non-diabetes } \\
(\mathrm{N}=\mathbf{2 4 4 7})\end{array}$ & $\begin{array}{l}\text { DMI } \\
(N=166)\end{array}$ & $\begin{array}{l}\text { DM2 } \\
(N=1497)\end{array}$ & $p$-value \\
\hline \multicolumn{5}{|l|}{ Transplant specific } \\
\hline Cyclosporine A & $129 \mid(52.8 \%)$ & $76(45.8 \%)$ & 727 (48.6\%) & 0.015 \\
\hline Tacrolimus & $84 I$ (34.4\%) & 74 (44.6\%) & $640(42.8 \%)$ & $<0.001$ \\
\hline Sirolimus & $201(8.2 \%)$ & $15(9.0 \%)$ & $129(8.6 \%)$ & 0.87 \\
\hline Mycophenolate mofetil & $1566(64.0 \%)$ & $96(57.8 \%)$ & $1018(68.0 \%)$ & 0.005 \\
\hline Azathioprine & 489 (20.0\%) & $35(21.1 \%)$ & $216(14.4 \%)$ & $<0.001$ \\
\hline Prednisone & 2245 (91.7\%) & $152(91.6 \%)$ & $|34|(89.6 \%)$ & 0.07 \\
\hline Calcineurin inhibitors $(\mathrm{Cl})$ & $2130(87.1 \%)$ & I 50 (90.4\%) & 1360 (90.9\%) & 0.001 \\
\hline Bone marrow suppressants (BMS) & $2042(83.5 \%)$ & $|3|(78.9 \%)$ & $1229(82.2 \%)$ & 0.21 \\
\hline $\mathrm{Cl}+\mathrm{BMS}+$ predn & 1597 (65.3\%) & $104(62.7 \%)$ & $1010(67.5 \%)$ & 0.24 \\
\hline $\mathrm{Cl}+\mathrm{BMS}+$ sirol & $24(1.0 \%)$ & $\mathrm{I}(0.6 \%)$ & $12(0.8 \%)$ & 0.87 \\
\hline Sirol+predn & $162(6.6 \%)$ & 14 (8.4\%) & $92(6.1 \%)$ & 0.50 \\
\hline $\mathrm{Cl}+$ sirol & $96(3.9 \%)$ & $9(5.4 \%)$ & $70(4.7 \%)$ & 0.39 \\
\hline $\mathrm{Cl}+$ sirol+predn & $69(2.8 \%)$ & $8(4.8 \%)$ & $46(3.1 \%)$ & 0.33 \\
\hline BMS+sirol & $116(4.7 \%)$ & $6(3.6 \%)$ & $61(4.1 \%)$ & 0.53 \\
\hline BMS+sirol+predn & $90(3.7 \%)$ & $5(3.0 \%)$ & $45(3.0 \%)$ & 0.51 \\
\hline $\mathrm{Cl}+\mathrm{BMS}+$ sirol+predn & $9(0.4 \%)$ & $0(0.0 \%)$ & $7(0.5 \%)$ & 0.63 \\
\hline \multicolumn{5}{|l|}{ Antihypertensives } \\
\hline Angiotensin-converting enzyme inhibitors & $77 \mathrm{I}(3 \mathrm{I} .5 \%)$ & $6 \mathrm{I}(36.7 \%)$ & $512(34.2 \%)$ & 0.11 \\
\hline Angiotensin receptor blockers & $282(11.5 \%)$ & $16(9.6 \%)$ & $250(16.7 \%)$ & $<0.001$ \\
\hline Beta blockers & 1357 (55.5\%) & $84(50.6 \%)$ & $878(58.7 \%)$ & 0.04 \\
\hline Calcium channel blockers & $810(33.1 \%)$ & $58(34.9 \%)$ & $568(37.9 \%)$ & 0.008 \\
\hline Diuretics & $625(25.5 \%)$ & $4 \mathrm{l}(24.7 \%)$ & $559(37.3 \%)$ & $<0.001$ \\
\hline Others & $230(9.4 \%)$ & $9(5.4 \%)$ & 165 (I I.0\%) & 0.04 \\
\hline \multicolumn{5}{|l|}{ Number of BP meds } \\
\hline Mean & $1.67 \pm 1.05$ & $1.62 \pm 1.08$ & $1.96 \pm 1.13$ & $<0.001$ \\
\hline Numbers & & & & $<0.001$ \\
\hline 0 & $333(13.6 \%)$ & 29 (I7.5\%) & $146(9.8 \%)$ & \\
\hline I & $770(31.5 \%)$ & $47(28.3 \%)$ & $375(25.1 \%)$ & \\
\hline 2 & $842(34.4 \%)$ & $54(32.5 \%)$ & $521(34.8 \%)$ & \\
\hline 3 & $398(16.3 \%)$ & 30 (18.1\%) & $321(21.4 \%)$ & \\
\hline 4 & $95(3.9 \%)$ & $6(3.6 \%)$ & $119(7.9 \%)$ & \\
\hline 5 & $7(0.3 \%)$ & $0(0.0 \%)$ & $14(0.9 \%)$ & \\
\hline 6 & $2(0.1 \%)$ & $0(0.0 \%)$ & I (0.1\%) & \\
\hline \multicolumn{5}{|l|}{ Lipid-lowering medications } \\
\hline HMG-CoA (statins) & 1205 (49.2\%) & 90 (54.2\%) & $86 \mid(57.5 \%)$ & $<0.001$ \\
\hline Others & 145 (5.9\%) & $7(4.2 \%)$ & $125(8.4 \%)$ & 0.005 \\
\hline \multicolumn{5}{|l|}{ Platelet specific } \\
\hline Aspirin & 817 (33.4\%) & $94(56.6 \%)$ & $783(52.3 \%)$ & $<0.001$ \\
\hline Others & $41(1.7 \%)$ & $9(5.4 \%)$ & $80(5.3 \%)$ & 0.001 \\
\hline Anticoagulants & I I (4.8\%) & $5(3.0 \%)$ & $78(5.2 \%)$ & 0.45 \\
\hline
\end{tabular}

Abbreviations: DM, diabetes mellitus; DMI, type I diabetes mellitus; DM2, type 2 diabetes mellitus; BP, blood pressure; predn, prednisone; sirol, sirolimus; HMG-CoA, 3-hydroxy-3-methylglutaryl-coenzyme A.

- The use of lipid-lowering medications and platelet-active medications was more common in the diabetic cohorts.

- Anticoagulant use was similar in all cohorts.

Tables 3 and 4 list medications by most common combinations used at baseline in this cohort. An increase in the diagnosis of diabetes is seen after transplantation because some forms of anti-immune therapy effect glucose metabolism. Tables 5 and 6 break down the relationship between graft vintage and immunomodulation therapies.

\section{Discussion}

In summary, the diabetic transplant recipients had a greater prevalence of PCVE and were more likely to have required multiple medications to control hypertension. They were also more likely to have received tacrolimus as opposed to cyclosporine than the non-diabetic transplant recipients.

In facilities familiar with post-transplant care, communication among subspecialists is usually successful; however, when frequent visits are required at relatively long distances, primary care internists must become progressively more 
Table 3 Demographic characteristics by immunotherapy medications

\begin{tabular}{|c|c|c|c|c|c|c|c|c|}
\hline $\begin{array}{l}\text { Baseline } \\
\text { medication } \\
\text { groups }\end{array}$ & $\begin{array}{l}\text { Cl+BMS+ } \\
\text { predn, } n=2695, \\
65.62 \%\end{array}$ & $\begin{array}{l}\text { Cl+predn, } \\
n=487 \\
I I .86 \%\end{array}$ & $\begin{array}{l}\text { BMS+predn, } \\
n=272,6.62 \%\end{array}$ & $\begin{array}{l}\text { CI+BMS, } \\
n=244,5.94 \%\end{array}$ & $\begin{array}{l}\text { BMS+sirol+ } \\
\text { predn, } n=\mid 24 \text {, } \\
3.02 \%\end{array}$ & $\begin{array}{l}\text { Cl+sirol+ } \\
\text { predn, } n=107, \\
2.61 \%\end{array}$ & $\begin{array}{l}\text { Others, } \\
n=178,4.33 \%\end{array}$ & $p$-value \\
\hline DM status & & & & & & & & 0.004 \\
\hline Non-DM & I 588 (58.9\%) & $292(60.0 \%)$ & 191 (70.2\%) & $|4|$ (57.8\%) & $8 \mathrm{I}(65.3 \%)$ & $60(56.1 \%)$ & $92(51.7 \%)$ & \\
\hline Type I DM & 104 (3.9\%) & 24 (4.9\%) & $9(3.3 \%)$ & $12(4.9 \%)$ & $5(4.0 \%)$ & $8(7.5 \%)$ & $4(2.2 \%)$ & \\
\hline Type 2 DM & 1003 (37.2\%) & I7I (35.1\%) & $72(26.5 \%)$ & 91 (37.3\%) & 38 (30.6\%) & 39 (36.4\%) & 82 (46.1\%) & \\
\hline Age & $51.46 \pm 9.35$ & $53.67 \pm 10.17$ & $52.15 \pm 9.12$ & $54.43 \pm 9.41$ & $50.69 \pm 9.00$ & $49.50 \pm 8.58$ & $52.79 \pm 8.82$ & $<0.001$ \\
\hline Non-White & $670(25.4 \%)$ & $90(19.1 \%)$ & 46 (17.2\%) & 51 (21.3\%) & $28(23.1 \%)$ & $23(21.9 \%)$ & 37 (21.3\%) & 0.008 \\
\hline Country & & & & & & & & $<0.001$ \\
\hline US & 1975 (73.3\%) & $326(66.9 \%)$ & I84 (67.6\%) & $186(76.2 \%)$ & 89 (7I.8\%) & 90 (84.1\%) & 147 (82.6\%) & \\
\hline Canada & 247 (9.2\%) & 135 (27.7\%) & $40(14.7 \%)$ & $40(16.4 \%)$ & II (8.9\%) & $6(5.6 \%)$ & $19(10.7 \%)$ & \\
\hline Brazil & 473 (I7.6\%) & $26(5.3 \%)$ & $48(17.6 \%)$ & $18(7.4 \%)$ & 24 (19.4\%) & II (10.3\%) & $12(6.7 \%)$ & \\
\hline Female & 975 (36.2\%) & 198 (40.7\%) & $102(37.5 \%)$ & $104(42.6 \%)$ & 44 (35.5\%) & $4 \mathrm{l}(38.3 \%)$ & 63 (35.4\%) & 0.30 \\
\hline Current smoker & $298(11.1 \%)$ & $43(8.8 \%)$ & $38(14.0 \%)$ & 28 (II.5\%) & II (8.9\%) & 12 (11.2\%) & 20 (II.2\%) & 0.49 \\
\hline Prevalent CVD & 521 (19.4\%) & $103(21.2 \%)$ & 56 (20.7\%) & 56 (23.2\%) & $25(20.3 \%)$ & $16(15.0 \%)$ & $43(24.2 \%)$ & 0.38 \\
\hline HTN & 2469 (91.6\%) & 460 (94.5\%) & $234(86.0 \%)$ & 221 (90.6\%) & $123(99.2 \%)$ & $103(96.3 \%)$ & $168(94.4 \%)$ & $<0.001$ \\
\hline $\begin{array}{l}\text { Graft vintage } \\
\text { (years) }\end{array}$ & $3.6[1.6,6.6]$ & $6.9[2.6,11.6]$ & $9.7[4.7,18.1]$ & $4.0[1.8,6.7]$ & $2.7[1.3,5.2]$ & $1.9[1.2,3.8]$ & $3.4[1.3,8.2]$ & $<0.001$ \\
\hline \multicolumn{9}{|l|}{ Graft type } \\
\hline Cadaver & 1567 (58.1\%) & $320(65.7 \%)$ & $129(47.4 \%)$ & $126(51.6 \%)$ & 67 (54.0\%) & 59 (55.1\%) & $100(56.2 \%)$ & $<0.001$ \\
\hline Living & 1111 (4I.2\%) & $160(32.9 \%)$ & $140(51.5 \%)$ & 117 (48.0\%) & 55 (44.4\%) & 47 (43.9\%) & $75(42.1 \%)$ & $<0.001$ \\
\hline BMI & $29.25 \pm 6.31$ & $28.45 \pm 5.82$ & $29.7 \mathrm{I} \pm 6.44$ & $29.24 \pm 5.81$ & $29.4 I \pm 6.15$ & $28.4 I \pm 5.59$ & $29.30 \pm 6.78$ & 0.10 \\
\hline Creatinine $(\mathrm{mg} / \mathrm{dL})$ & $1.61 \pm 0.46$ & $1.70 \pm 0.48$ & $1.7 I \pm 0.57$ & $1.53 \pm 0.41$ & $1.77 \pm 0.49$ & $1.75 \pm 0.51$ & $1.61 \pm 0.53$ & $<0.001$ \\
\hline eGFR & $49.88 \pm 16.33$ & $44.91 \pm 15.23$ & $46.44 \pm 16.50$ & $49.68 \pm 14.43$ & $44.35 \pm 14.85$ & $44.8 I \pm 14.98$ & $50.23 \pm 17.23$ & $<0.001$ \\
\hline CKD & & & & & & & & $<0.001$ \\
\hline $\mathrm{GRF} \geq 90$ & $53(2.0 \%)$ & $6(1.3 \%)$ & $3(1.1 \%)$ & $\mathrm{I}(0.4 \%)$ & $\mathrm{I}(0.8 \%)$ & $2(1.9 \%)$ & $3(1.7 \%)$ & \\
\hline $60 \leq \mathrm{GFR}<90$ & $577(21.8 \%)$ & 71 (15.1\%) & 46 (I7.2\%) & $55(23.0 \%)$ & 14 (1 I.6\%) & II (10.5\%) & $44(25.3 \%)$ & \\
\hline $30 \leq \mathrm{GFR}<60$ & 1797 (68.0\%) & $318(67.7 \%)$ & $178(66.4 \%)$ & $166(69.5 \%)$ & 87 (71.9\%) & 81 (77.1\%) & $110(63.2 \%)$ & \\
\hline $15 \leq$ GFR $8<30$ & $214(8.1 \%)$ & $75(16.0 \%)$ & $40(14.9 \%)$ & $17(7.1 \%)$ & $19(15.7 \%)$ & II (I0.5\%) & $17(9.8 \%)$ & \\
\hline GFR $<15$ & $0(0.0 \%)$ & $0(0.0 \%)$ & I $(0.4 \%)$ & $0(0.0 \%)$ & $0(0.0 \%)$ & $0(0.0 \%)$ & $0(0.0 \%)$ & \\
\hline $\begin{array}{l}\text { Cholesterol } \\
(\mathrm{mg} / \mathrm{dL})\end{array}$ & $183.33 \pm 42.26$ & $181.95 \pm 36.82$ & $180.80 \pm 45.39$ & $169.02 \pm 40.43$ & $214.88 \pm 53.30$ & $222.66 \pm 61.06$ & $194.08 \pm 49.07$ & $<0.001$ \\
\hline $\begin{array}{l}\text { Triglycerides } \\
(\mathrm{mg} / \mathrm{dL})\end{array}$ & $189.68 \pm 123.74$ & $191.62 \pm 117.57$ & $189.22 \pm 173.82$ & $|89.34 \pm| 19.48$ & $292.34 \pm 197.81$ & $367.90 \pm 776.16$ & $218.71 \pm 165.98$ & $<0.001$ \\
\hline $\mathrm{HDL}(\mathrm{mg} / \mathrm{dL})$ & $46.43 \pm 13.92$ & $47.52 \pm 14.27$ & $45.77 \pm 13.84$ & $41.88 \pm 13.15$ & $45.34 \pm 12.95$ & $49.77 \pm \mid 3.81$ & $44.91 \pm 13.72$ & $<0.001$ \\
\hline LDL (mg/dL) & $|00.29 \pm 33.7|$ & $98.42 \pm 30.58$ & $98.04 \pm 30.86$ & $91.19 \pm 32.79$ & $|17.33 \pm 4| .39$ & II $15.09 \pm 37.48$ & $107.04 \pm 38.12$ & $<0.001$ \\
\hline Homocysteine & $|00.29 \pm 33.7|$ & $98.42 \pm 30.58$ & $98.04 \pm 30.86$ & $91.19 \pm 32.79$ & $\mid 17.33 \pm 41.39$ & II5.09₫37.48 & $107.04 \pm 38.12$ & 0.49 \\
\hline
\end{tabular}

Abbreviations: DM, diabetes mellitus; DMI, type I diabetes mellitus; DM2, type 2 diabetes mellitus; CVD, cardiovascular disease; BMI, body mass index; eGFR, estimated glomerular filtration rate; CKD, chronic kidney disease; HTN, hypertension; HDL, high-density lipoprotein; LDL, low-density lipoprotein; Cl, calcineurin inhibitors (cyclosporine, tacrolimus); BMS, bone marrow suppressants (azathioprine, mycophenolate); sirol, mTOR inhibitor (sirolimus); predn, antilymphocytics (prednisone); mTOR, mechanistic target of rapamycin.

familiar with the interplay of immunosuppressive agents, antihypertensive medications, and new drugs for diabetes.

This study design directs attention to information on risk after the initial post-transplant period of instability, infection, and rejection. In this study, among the 4110 randomized participants, $38.9 \%$ had diabetes and $19.5 \%$ had previous cardiovascular events that had not precluded organ transplant. As such, it is quite representative of the stable renal transplant population that is seen in most transplant and nephrology clinics. The FAVORIT database is ideal for analysis as it has information on comorbid conditions, medications, and outcomes of interest that other databases may not have. This and other registries permit us to focus on the increasing population for whom successful organ transplantation has permitted a life free from dialysis. Yet long-term risks of cardiovascular and non-cardiovascular morbidity remain to a certain extent related to comorbidities such as underlying cardiovascular disease, hypertension, diabetes, underlying renal disease, and effects of immunosuppression. Patients undergoing renal allograft transplantation for polycystic disease may not experience recurrence of this condition in their kidney allograft, but individuals with diabetes, focal segmental glomerulosclerosis, membranous or immunoglobulin A nephropathy, and lupus, amyloid, or 
Table 4 Demographic characteristics by immunotherapy medications (row wise)

\begin{tabular}{|c|c|c|c|c|c|c|c|c|}
\hline $\begin{array}{l}\text { Baseline } \\
\text { medication groups }\end{array}$ & $\begin{array}{l}\text { Cl+BMS+predn, } \\
n=2695,65.62 \%\end{array}$ & $\begin{array}{l}\text { Cl+predn, } \\
\text { n=487, } \\
I I .86 \%\end{array}$ & $\begin{array}{l}\text { BMS+predn, } \\
n=272,6.62 \%\end{array}$ & $\begin{array}{l}\text { Cl+BMS, } \\
\mathrm{n}=244, \\
5.94 \%\end{array}$ & $\begin{array}{l}\text { BMS+sirol+predn, } \\
n=124,3.02 \%\end{array}$ & $\begin{array}{l}\text { Cl+sirol+predn, } \\
n=107,2.61 \%\end{array}$ & $\begin{array}{l}\text { Others, } \\
n=178, \\
4.33 \%\end{array}$ & $p$-value \\
\hline DM status & & & & & & & & 0.004 \\
\hline Non-DM & 1588 (64.9\%) & 292 (II.9\%) & I9| (7.8\%) & $|4|$ (5.8\%) & 81 (3.3\%) & $60(2.5 \%)$ & 92 (3.8\%) & \\
\hline Type I DM & 104 (62.7\%) & $24(14.5 \%)$ & 9 (5.4\%) & $12(7.2 \%)$ & $5(3.0 \%)$ & $8(4.8 \%)$ & $4(2.4 \%)$ & \\
\hline Type 2 DM & $1003(67.0 \%)$ & I7| (II.4\%) & 72 (4.8\%) & 91 (6.1\%) & 38 (2.5\%) & $39(2.6 \%)$ & 82 (5.5\%) & \\
\hline Non-White & 670 (70.9\%) & $90(9.5 \%)$ & 46 (4.9\%) & 51 (5.4\%) & $28(3.0 \%)$ & 23 (2.4\%) & 37 (3.9\%) & \\
\hline Country & & & & & & & & $<0.001$ \\
\hline US & 1975 (65.9\%) & $326(10.9 \%)$ & $184(6.1 \%)$ & 186 (6.2\%) & 89 (3.0\%) & $90(3.0 \%)$ & 147 (4.9\%) & \\
\hline Canada & 247 (49.6\%) & 135 (27.1\%) & 40 (8.0\%) & 40 (8.0\%) & II (2.2\%) & $6(1.2 \%)$ & 19 (3.8\%) & \\
\hline Brazil & 473 (77.3\%) & $26(4.2 \%)$ & 48 (7.8\%) & 18 (2.9\%) & 24 (3.9\%) & II (I.8\%) & $12(2.0 \%)$ & \\
\hline Female & 975 (63.9\%) & $198(13.0 \%)$ & $102(6.7 \%)$ & $104(6.8 \%)$ & 44 (2.9\%) & $41(2.7 \%)$ & $63(4.1 \%)$ & \\
\hline Current smoker & $298(66.2 \%)$ & $43(9.6 \%)$ & $38(8.4 \%)$ & $28(6.2 \%)$ & II (2.4\%) & $12(2.7 \%)$ & 20 (4.4\%) & \\
\hline Prevalent CVD & 521 (63.5\%) & $103(12.6 \%)$ & 56 (6.8\%) & $56(6.8 \%)$ & $25(3.0 \%)$ & $16(2.0 \%)$ & 43 (5.2\%) & \\
\hline $\mathrm{HTN}$ & 2469 (65.4\%) & 460 (12.2\%) & 234 (6.2\%) & 221 (5.8\%) & $123(3.3 \%)$ & $103(2.7 \%)$ & 168 (4.4\%) & \\
\hline Graft type & & & & & & & & $<0.001$ \\
\hline Cadaver & 1567 (66.2\%) & $320(13.5 \%)$ & $129(5.4 \%)$ & 126 (5.3\%) & 67 (2.8\%) & $59(2.5 \%)$ & 100 (4.2\%) & \\
\hline Living & IIII (65.2\%) & $160(9.4 \%)$ & 140 (8.2\%) & 117 (6.9\%) & 55 (3.2\%) & $47(2.8 \%)$ & 75 (4.4\%) & \\
\hline \multicolumn{9}{|l|}{ CKD } \\
\hline $\mathrm{GRF} \geq 90$ & $53(76.8 \%)$ & $6(8.7 \%)$ & $3(4.3 \%)$ & I (I.4\%) & I (I.4\%) & 2 (2.9\%) & $3(4.3 \%)$ & \\
\hline $60 \leq \mathrm{GFR}<90$ & 577 (70.5\%) & $71(8.7 \%)$ & $46(5.6 \%)$ & $55(6.7 \%)$ & 14 (1.7\%) & II (I.3\%) & $44(5.4 \%)$ & \\
\hline $30 \leq \mathrm{GFR}<60$ & 1797 (65.7\%) & $318(11.6 \%)$ & 178 (6.5\%) & 166 (6.1\%) & 87 (3.2\%) & 81 (3.0\%) & 110 (4.0\%) & \\
\hline $15 \leq$ GFR $8<30$ & $214(54.5 \%)$ & 75 (19.1\%) & 40 (10.2\%) & 17 (4.3\%) & 19 (4.8\%) & II (2.8\%) & 17 (4.3\%) & \\
\hline GFR $<15$ & $0(0.0 \%)$ & $0(0.0 \%)$ & I (100.0\%) & $0(0.0 \%)$ & $0(0.0 \%)$ & $0(0.0 \%)$ & $0(0.0 \%)$ & \\
\hline
\end{tabular}

Abbreviations: DM, diabetes mellitus; DMI, type I diabetes mellitus; DM2, type 2 diabetes mellitus; CVD, cardiovascular disease; BMI, body mass index; eGFR, estimated glomerular filtration rate; CKD, chronic kidney disease; Cl, calcineurin inhibitors (cyclosporine, tacrolimus); BMS, bone marrow suppressants (azathioprine, mycophenolate); sirol, mTOR inhibitor (sirolimus); predn, antilymphocytics (prednisone); mTOR, mechanistic target of rapamycin; HTN, hypertension;.

hemolytic uremic syndrome may have disease recurrence. In addition, several of the immunosuppressant regimens used are "diabetogenic" and the development of steroid-dependent diabetes is not uncommon and may impact prognosis. ${ }^{8}$ This information must be available to the clinicians who will treat transplant patients.

Diabetic populations are at higher risk of all-cause mortality than non-diabetic populations. The addition of immunosuppression to prevent rejection of a solid organ magnifies this excess risk. Taking these facts into account, we focused on the treatment of diabetes, hypertension, and immunosuppression at baseline of an international trial enrolling stable renal allograft patients in both North and South America. When analyzed from the perspective of non-diabetic or type 1 or type 2 diabetic cohorts, we found baseline renal allograft function to be similar. There was clearly an excess of previous cardiovascular events in the diabetic patients as well as more use of statins with lower lipid risk profiles. In addition, requirement of more medications needed to treat hypertension in the diabetic cohorts was evident. Given such findings, one would anticipate an excess cardiovascular risk in the diabetic cohort.

The observation of this trial that the diabetic cohort was more likely to have received tacrolimus as opposed to cyclosporine than the non-diabetic cohort may be due to the reduced effect that cyclosporine has on glucose metabolism in patients already under treatment for diabetes. This study reflects an era in which cyclosporine use was diminishing in diabetic cohorts, especially in those undergoing combined simultaneous kidney-pancreas transplantation. ${ }^{9}$ Although it is likely that the migration to tacrolimus was due to perceived improved efficacy, it is possible that tacrolimus was preferred to cyclosporine in the diabetic cohort to avoid hyperkalemia and arteriolar hyaline thickening/obliterative disease. ${ }^{10,11}$ Cyclosporine use has diminished since that time due to concern for allograft nephropathy. ${ }^{12-14}$ Comparative observations of diabetes vs non-diabetes use have not previously been reported.

There exists a significant body of research linking insulin resistance and hypertension therapy resistance. The observation that in stable renal transplant population, hypertension therapy for diabetic patients may require more complex drug regimens is similar to those noted in both single-center and multinational trials enrolling patients with diabetes and renal disease. ${ }^{15,16}$ Our study confirms these reports and demonstrates that type 2 diabetic patients required significantly greater numbers of antihypertensives than non-diabetic patients of the similar age (Table 2). One could postulate that among diabetic transplant recipients with relatively resistant 
Table 5 Relationship between graft vintage and immunotherapy

\begin{tabular}{|c|c|c|c|c|}
\hline \multirow[t]{3}{*}{ Graft vintage } & Tercile I & Tercile 2 & Tercile 3 & $p$-value \\
\hline & $N=1373$ & $N=1357$ & $N=1360$ & \\
\hline & $0.34-2.25$ & $2.26-6.12$ & $6.13-46.18$ & \\
\hline \multicolumn{5}{|l|}{ Transplant specific } \\
\hline Cyclosporine A & 477 (34.7\%) & 675 (49.7\%) & 929 (68.3\%) & $<0.001$ \\
\hline Tacrolimus & 797 (58.0\%) & $552(40.7 \%)$ & 204 (I5.0\%) & $<0.001$ \\
\hline Sirolimus & 172 (I2.5\%) & $120(8.8 \%)$ & $51(3.8 \%)$ & $<0.001$ \\
\hline Mycophenolate mofetil & $1065(77.6 \%)$ & $1009(74.4 \%)$ & $599(44.0 \%)$ & $<0.001$ \\
\hline Azathioprine & $108(7.9 \%)$ & $464(34.1 \%)$ & 161 (11.9\%) & $<0.001$ \\
\hline Prednisone & 1237 (90.1\%) & $1228(90.5 \%)$ & $1255(92.3 \%)$ & 0.047 \\
\hline Calcineurin inhibitors $(\mathrm{Cl})$ & $1268(92.4 \%)$ & $1226(90.4 \%)$ & II3I (83.2\%) & $<0.001$ \\
\hline Bone marrow suppressants (BMS) & 1168 (85.1\%) & II 68 (86.1\%) & 1052 (77.4\%) & $<0.001$ \\
\hline Group medication & & & & $<0.001$ \\
\hline $\mathrm{Cl}+\mathrm{BMS}+$ predn & $972(70.8 \%)$ & $953(70.3 \%)$ & $76 \mid(56.0 \%)$ & \\
\hline $\mathrm{Cl}+$ predn & $108(7.9 \%)$ & $117(8.6 \%)$ & $258(19.0 \%)$ & \\
\hline BMS+predn & $30(2.2 \%)$ & 57 (4.2\%) & $182(13.4 \%)$ & \\
\hline $\mathrm{Cl}+\mathrm{BMS}$ & 79 (5.8\%) & 95 (7.0\%) & $69(5.1 \%)$ & \\
\hline BMS+sirol+predn & 51 (3.7\%) & 49 (3.6\%) & $23(1.7 \%)$ & \\
\hline $\mathrm{Cl}+$ sirol+predn & 59 (4.3\%) & 37 (2.7\%) & 10 (0.7\%) & \\
\hline Others & 74 (5.4\%) & 48 (3.5\%) & 56 (4.1\%) & \\
\hline
\end{tabular}

Abbreviations: sirol, mTOR inhibitor (sirolimus); predn, antilymphocytics (prednisone); mTOR, mechanistic target of rapamycin.

Table 6 Relationship between graft vintage and immunotherapy (row wise)

\begin{tabular}{|c|c|c|c|c|}
\hline Graft vintage & $\begin{array}{l}\text { Tercile I } \\
\mathrm{N}=1373 \\
0.34-2.25\end{array}$ & $\begin{array}{l}\text { Tercile } 2 \\
N=1357 \\
2.26-6.12\end{array}$ & $\begin{array}{l}\text { Tercile } 3 \\
N=1360 \\
6.13-46.18\end{array}$ & $p$-value \\
\hline \multicolumn{5}{|l|}{ Transplant specific } \\
\hline Cyclosporine A & 477 (22.9\%) & $675(32.4 \%)$ & $929(44.6 \%)$ & 0.001 \\
\hline Tacrolimus & 797 (5I.3\%) & $552(35.5 \%)$ & $204(13.1 \%)$ & \\
\hline Sirolimus & $172(50.1 \%)$ & 120 (35.0\%) & $51(14.9 \%)$ & \\
\hline Mycophenolate mofetil & $1065(39.8 \%)$ & $1009(37.7 \%)$ & $599(22.4 \%)$ & \\
\hline Azathioprine & $108(14.7 \%)$ & 161 (22.0\%) & $464(63.3 \%)$ & \\
\hline Prednisone & 1237 (33.3\%) & $1228(33.0 \%)$ & 1255 (33.7\%) & \\
\hline Calcineurin inhibitors $(\mathrm{Cl})$ & $1268(35.0 \%)$ & $1226(33.8 \%)$ & II3I (3I.2\%) & \\
\hline Bone marrow suppressants (BMS) & 1168 (34.5\%) & I I 68 (34.5\%) & 1052 (31.1\%) & \\
\hline Group medication & & & & 0.001 \\
\hline $\mathrm{Cl}+\mathrm{BMS}+$ predn & $972(36.2 \%)$ & $953(35.5 \%)$ & 761 (28.3\%) & \\
\hline $\mathrm{Cl}+$ predn & $108(22.4 \%)$ & II (24.2\%) & $258(53.4 \%)$ & \\
\hline BMS+predn & $30(11.2 \%)$ & $57(21.2 \%)$ & I $82(67.7 \%)$ & \\
\hline $\mathrm{Cl}+\mathrm{BMS}$ & 79 (32.5\%) & 95 (39.1\%) & $69(28.4 \%)$ & \\
\hline BMS+sirol+predn & $5 \mathrm{I}(4 \mathrm{I} .5 \%)$ & 49 (39.8\%) & $23(18.7 \%)$ & \\
\hline $\mathrm{Cl}+$ sirol+predn & 59 (55.7\%) & 37 (34.9\%) & 10 (9.4\%) & \\
\hline Others & 74 (4I.6\%) & $48(27.0 \%)$ & 56 (31.5\%) & \\
\hline
\end{tabular}

Abbreviations: sirol, mTOR inhibitor (sirolimus); predn, antilymphocytics (prednisone); mTOR, mechanistic target of rapamycin.

hypertension, a medication solely targeting insulin resistance, or the causes of such resistance, might also reduce the need for complex antihypertensive therapy. Further study and use of biguanides in the type 2 diabetic cohort appears appropriate if transplant function is adequate. ${ }^{17}$

Although a reasonably low-risk group of patients with diabetes and renal disease can be identified and treated with successful renal transplant, recipients with diabetes demonstrated an excess risk for both cardiovascular and infectious events when compared to non-diabetic populations. ${ }^{18-21}$ Successful prediction of subsequent cardiovascular events (SCVE) from a history of PCVE has been demonstrated for diabetic patients with impaired renal function. ${ }^{22}$ In a small study of diabetic patients on long-term dialysis, we were unable to demonstrate this predictability; however, the populations were quite different..$^{23}$ The current analysis adds to the literature a cross-sectional evaluation from an international trial demonstrating some of the differences in therapies 
chosen for stable renal allograft recipients depending upon the absence, presence, and type of diabetes, and PCVE. In summary, the diabetic transplant recipients had a greater prevalence of PCVE and were more likely to have required multiple medications to control hypertension ${ }^{24,25}$ and were also more likely to have received tacrolimus as opposed to cyclosporine than the non-diabetic transplant recipients. ${ }^{26-28}$

It would be anticipated that long-term stability of transplant function would allow for a decrease in antirejection therapy to the minimum required to avoid side effects from therapy. This is confirmed in this study (Tables 5 and 6) by the progressive reduction in prevalence of calcineurin inhibitors and bone marrow suppressant usage as allograft vintage increased. The strong association observed between renal allograft vintage and immunomodulation therapy evident in this study may explain, in part, some differences between our non-diabetic and diabetic cohorts (as indicated in Table 1). Renal replacement therapy occurs earlier in type 1 diabetic cohorts and may be associated with a need for pancreatic transplant. There were no patients enrolled in this trial with simultaneous pancreatic-kidney allografts. By age $>50$ years, it would be anticipated that a larger percentage of the diabetic cohort than the non-diabetic cohort would have undergone more than one transplant.

Our analysis has several limitations. Type 2 diabetes mellitus in this renal transplant recipient cohort may be a somewhat heterogeneous descriptive given the possibility that this diagnosis may be related to required use of immunosuppression. $\mathrm{C}$ peptide measurement was not undertaken as part of the study. Some of the diabetes identified may have been post-transplant and records of the duration of diabetes were not available. ${ }^{29-31}$ Although "new onset diabetes" in the face of immunosuppression is considered by many to have less impact on cardiovascular events than longer term diabetes in transplant populations, studies of "new onset diabetes" in patients with heart failure and coronary events do not have a less severe prognosis. ${ }^{32,33}$ Further studies on the impact of new onset diabetes on outcomes would be appropriate. In our study, $90 \%$ of the patients with diabetes had at least 1 year of diabetes. Although duration of dialysis was not available in the database of the current report, two reports indicate that since 2000, the impact of dialysis duration on transplant allograft survival has decreased substantially. ${ }^{34,35}$ It is understood that populations with diabetes mellitus have a higher prevalence of cardiovascular and non-cardiovascular morbidity and mortality than populations without diabetes. The excess risk is due to relative native immunosuppression. Underappreciated sources of excess risk may be cardiac autonomic dysfunction ${ }^{36}$ and peripheral neuropathy that interact with blood pressure and kidney function. The current study is unable to assess the impact of these effects in this population. Another limitation of this and other studies is a lack of information regarding prior renal allografts, vascular access procedures, or duration of dialysis. It is quite likely that, although the groups were of similar ages, the type 1 diabetic patients had prior renal allografts that had failed and this may have influenced subsequent therapy. The current presentation is not an outcome study, and differences in treatment may or may not impact outcomes due to extensive screening pretransplantation and due to selection bias for entry into the folic acid/vitamin B12 treatment trial. Likewise, this analysis cannot inform us on immunotherapy for diabetic patients during end-stage renal disease or for those who do not survive the early postoperative course. The enrollment period for this study spanned a 5-year period, during which immunomodulation transitioned to favor tacrolimus use. There was regional (country) variation in both tacrolimus use and prevalence of type 2 diabetes.

Stable diabetic renal transplant recipients had a greater prevalence of PCVE, were more likely to have required multiple medications to control hypertension, and were also more likely to have received tacrolimus as opposed to cyclosporine than the non-diabetic transplant recipients. Among stable renal transplant recipients, there were also differences in immunomodulatory therapy based upon graft vintage, which was different for type 1 and type 2 diabetics than for non-diabetics.

The effect of differences in treatment between nondiabetic and diabetic cohorts and variations in therapy based upon graft vintage after stable renal transplantation upon outcomes have not yet been studied, which could provide additional information that might lead to improved care.

\section{Author contributions}

All authors contributed toward data analysis, drafting and critically revising the paper and agree to be accountable for all aspects of the work.

\section{Disclosure}

The authors report no conflicts of interest in this work.

\section{References}

1. Weinrauch L, D'Elia J, Healy R, et al. Asymptomatic coronary artery disease: angiography in diabetic patients before renal transplantation: a relationship of findings to post-operative survival. Ann Intern Med. 1978;88(3):346-348. 
2. D'Elia JA, Weinrauch LA, Kaldany A, et al. Improving survival following renal transplantation for diabetics with severe coronary artery disease. Diabetes Care. 1980;4(3):380-382.

3. Weinrauch LA, D'Elia JA, Monaco AP, et al. Preoperative evaluation for diabetic renal transplantation: impact of clinical, laboratory, and echocardiographic parameters on patient and allograft survival. Am J Med. 1992;93(1):19-28.

4. Manske CL, Wang Y, Rector T, Wilson RF, White CW. Coronary revascularisation in insulin-dependent diabetic patients with chronic renal failure. Lancet. 1992;340(8826):998-1002

5. Bostom AG, Carpenter MA, Hunsicker L, et al; FAVORIT Study Investigators. Baseline characteristics of participants in the Folic Acid for Vascular Outcome Reduction in Transplantation (FAVORIT) Trial. Am J Kidney Dis. 2009;53(1):121-128.

6. Bostom AG, Carpenter MA, Kusek JW, et al. Homocysteine-lowering and cardiovascular disease outcomes in kidney transplant recipients: primary results from the folic acid for vascular outcome reduction in transplantation trial. Circulation. 2011;123(16):1763-1770.

7. Czyzyk A, Królewski AS. Is cigarette smoking more frequent among insulin-treated diabetics? Diabetes. 1976;25(8):717-718.

8. Kasiske BL, Snyder JJ, Gilbertson D, Matas AJ. Diabetes mellitus after kidney transplantation in the United States. Am J Transplant. 2003;3(2):178-185.

9. Sampaio MS, Kuo HT, Bunnapradist S. Outcomes of simultaneous pancreas-kidney transplantation in type 2 diabetic recipients. Clin J Am Soc Nephrol. 2011;6(5):1198-1206.

10. Morozumi K, Takeda A, Uchida K, Mihatsch MJ. Cyclosporine nephrotoxicity: how does it affect renal allograft function and transplant morphology? Transplant Proc. 2004;36(2 Suppl):S251-S256.

11. Kambham N, Nagarajan S, Shah S, Li L, Salvatierra O, Sarwal MM. A novel, semiquantitative, clinically correlated calcineurin inhibitor toxicity score for renal allograft biopsies. Clin J Am Soc Nephrol. 2007:2(1):135-142.

12. Jevnikar A, Arlen D, Barrett B, et al. Five-year study of tacrolimus as secondary intervention versus continuation of cyclosporine in renal transplant patients at risk for chronic renal allograft failure. Transplantation. 2008;86(7):953-960.

13. Jurewicz WA. Tacrolimus versus cyclosporin immunosuppression: long-term outcome in renal transplantation. Nephrol Dial Transplant 2003;18(Suppl 1):i7-i11.

14. Ekberg H, Tedesco-Silva H, Demirbas A, et al; ELITE-Symphony Study. Reduced exposure to calcineurin inhibitors in renal transplantation. N Engl J Med. 2007;357(25): 2562-2575.

15. Weinrauch LA, Bayliss G, Segal AR, Liu J, Wisniewski E, D’Elia JA. Renal function alters antihypertensive regimens in type 2 diabetic patients. J Clin Hypertens (Greenwich). 2016;18(9):878-883.

16. Weinrauch LA, D’Elia J, Finn PV, et al. Strategies for glucose control in a study population with diabetes, renal disease and anemia (TREAT study). Diabetes Res Clin Pract. 2016;113:143-151.

17. Weinrauch LA, Bayliss G, Segal AR, Liu J, Wisniewski E, D'Elia JA Treating hypertension in diabetic patients with advanced chronic kidney disease: what should we have in mind? J Clin Hypertens (Greenwich). 2016;18(10):1077-1078.

18. Hart A, Weir MR, Kasiske BL. Cardiovascular risk assessment in kidney transplantation. Kidney Int. 2015; 87(3):527-534.

19. Lentine KL, Costa SP, Weir MR, et al; American Heart Association Council on the Kidney in Cardiovascular Disease and Council on Peripheral Vascular Disease. Cardiac disease evaluation and management among kidney and liver transplantation candidates: a scientific statement from the American Heart Association and the American College of Cardiology Foundation. JAm Coll Cardiol. 2012;60(5):434-480.
20. Maamoun HAH, Soliman AR, Fathy A, Elkhatib M, Shaheen N. Diabetes mellitus as predictor of patient and graft survival after kidney transplantation. Transplant Proc. 2013;45(9):3245-3248.

21. Revanur KR, Jardine AG, Kingsmore DB, Jaques BC, Hamilton DH, Jindal RM. Influence of diabetes mellitus on patient and graft survival in recipients of kidney transplantation. Clin Transplant. 2001; 15(2):89-94

22. Charytan DM, Lewis EF, Desai AS, et al. Cause of death in patients with diabetic CKD enrolled in the Trial to Reduce Cardiovascular Events With Aranesp Therapy (TREAT). Am J Kidney Dis. 2015;66(3): 429-440.

23. Bayliss GP, Weinrauch LA, Gleason RE, Lee AT, D'Elia JA. Do biologic markers predict cardiovascular endpoints in diabetic end stage renal disease? A prospective longitudinal study. Clin Kidney J. 2013;6(6):599-603.

24. Weir MR, Townsend RR. Refractory hypertension: an important clinical phenotype. Hypertension. 2014;63(3):447-448.

25. Carpenter MA, Weir MR, Adey DB, House AA, Bostom AG, Kusek JW. Inadequacy of cardiovascular risk factor management in chronic kidney transplantation - evidence from the FAVORIT study. Clin Transplant. 2012;26(4):E438-E446.

26. Weir MR, Pearson TC, Patel A, et al. Long-term follow-up of kidney transplant recipients in the Spare-the-Nephron-Trial. Transplantation. 2017;101(1):157-165.

27. Vincenti F, Friman S, Scheuermann E; DIRECT (Diabetes Incidence after Renal Transplantation: Neoral C Monitoring Versus Tacrolimus) Investigators. Results of an international, randomized trial comparing glucose metabolism disorders and outcome with cyclosporine versus tacrolimus. Am J Transplant. 2007;7(6):1506-1514

28. Waid T; CRAF Study Group. Tacrolimus as secondary intervention vs. cyclosporine continuation in patients at risk for chronic renal allograft failure. Clin Transplant. 2005;19(5):573-580.

29. Choi JY, Kwon OJ. Post-transplant diabetes mellitus: is it associated with poor allograft outcomes in renal transplants? Transplant Proc. 2013;45(8):2892-2898.

30. Tufton N, Ahmad S, Rolfe C, Rajkariar R, Byrne C, Chowdhury TA. New onset diabetes after renal transplantation. Diabet Med. 2014;31(11): 1284-1292.

31. Cotovio P, Neves M, Rodrigues L, et al. New-onset diabetes after transplantation: assessment of risk factors and clinical outcomes. Transplant Proc. 2013;45(3):1079-1083.

32. Aguilar D, Solomon SD, Køber L, et al. Newly diagnosed and previously known diabetes mellitus and 1-year outcomes of acute myocardial infarction: the VALsartan In Acute myocardial iNfarcTion (VALIANT) trial. Circulation. 2004;110(12):1572-1578.

33. Ertelt K, Brener SJ, Mehran R, Ben-Yehuda O, McAndrew T, Stone GW. Comparison of outcomes and prognosis of patients with versus without newly diagnosed diabetes mellitus after primary percutaneous coronary intervention for ST-elevation myocardial infarction (the HORIZONSAMI Study). Am J Cardiol. 2017;119(12):1917-1923.

34. Helentera I, Salmela K, KyllonenI, Koskinen P, Gronhagen-Riska C, Finne P. Pretransplant dialysis duration and risk of death after kidney transplantation in the current era. Transplantation. 2014;98(4): 458-464.

35. Haller MC, Kainz A, Baer H, Oberbauer R. Dialysis vintage and outcomes after kidney transplantation: a retrospective study. Clin JAm Soc Nephrol. 2016;12(1):122-130.

36. Weinrauch LA, Kennedy FP, Gleason RE, Keough J, D'Elia JA. Relationship between autonomic function and the progression of renal disease in diabetic proteinuria: implications for blood pressure control. Am J Hypertension. 1998;11(3 Pt 1):302-308. 


\section{Publish your work in this journal}

The International Journal of Nephrology and Renovascular Disease is an international, peer-reviewed open access journal focusing on the pathophysiology of the kidney and vascular supply. Epidemiology, screening, diagnosis, and treatment interventions are covered as well as basic science, biochemical and immunological studies. The manuscript management system is completely online and includes a very quick and fair peer-review system, which is all easy to use. Visit http://www. dovepress.com/testimonials.php to read real quotes from published authors.

Submit your manuscript here: https://www.dovepress.com/international-journal-of-nephrology-and-renovascular-disease-journal 